\title{
Prevalence of Antenatal Depression and Associated Factors among Pregnant Women Attending Antenatal Care at Health Institutions of Faafan Zone, Somali Region, Eastern Ethiopia
}

\author{
Edao Tesa Keliyo ${ }^{1},{ }^{1}$ Meka Kedir Jibril, ${ }^{1}$ and Girma Tadesse Wodajo ${ }^{2}$ \\ ${ }^{1}$ Department of Nursing College of Medicine and Health Science, Jigjiga University, Jigjiga, Ethiopia \\ ${ }^{2}$ Department of Public Health College of Medicine and Health Science, Jigjiga University, Jigjiga, Ethiopia \\ Correspondence should be addressed to Edao Tesa Keliyo; edao_tesa@yahoo.com
}

Received 18 January 2020; Accepted 15 August 2021; Published 30 August 2021

Academic Editor: Giovanni Galeoto

Copyright (c) 2021 Edao Tesa Keliyo et al. This is an open access article distributed under the Creative Commons Attribution License, which permits unrestricted use, distribution, and reproduction in any medium, provided the original work is properly cited.

\begin{abstract}
Background. Depression is a common global mental health tragedy which affects more than 30 million people of all ages. Antenatal depression is higher among low-income countries where maternal and psychosocial factors act as determinant factors for its occurrence. Aim. This study is aimed at assessing the prevalence of antenatal depression and its associated factors among pregnant women attending health institutions of Faafan zone of Somali regional state, Eastern Ethiopia. Method. An institutional-based cross-sectional study design was conducted among randomly selected 403 pregnant women from January to September 2015. EPDS with 13 cutoff points was used to screen antenatal depression. Bivariate and multivariate logistic regressions were used to identify associated factors. Result. The study showed that $24.3 \%$ of women had antenatal depression. Marital status, educational status, chronic medical illness, previous depression history, and social support were factors associated with antenatal depression. Conclusion. The study revealed that the prevalence of antenatal depression was 24.3\%. Ethiopia Federal Ministry of Health and Somali Regional Health Bureau should work very hard to create awareness on the importance of pregnancy planning and social support during pregnancy.
\end{abstract}

\section{Introduction}

Depression is a common global mental health tragedy, which can manifest with depressed mood, feeling of guilt, loss of interest, lack of sleep, and low self-esteem. Globally, it affects more than 30 million people of all ages [1]. By 2030, depression is predicted to be the second leading cause of morbidity in developing countries. A systematic review study indicated that antenatal depression is higher among low-income countries along with maternal and psychosocial factors as determinant factors for its occurrence [2, 3]. Prenatal depression encompasses major and minor depressive episodes beginning during pregnancy which can be characterized by antenatal depression symptoms like depressed mood, anxiety, poor fetal attachment, loss of concentration, and feelings of low self- esteem $[4,5]$. Depression is the leading disease for women between the ages of 15 and 44 years in both high- and lowand middle-income countries [6]. Depression is currently the leading cause of nonfatal burden when considering all mental and physical illnesses, accounting for approximately $10 \%$ of total years lived with disability in low- and middle-income countries. By 2030, depression alone is likely to be the third leading cause of disease burden in low-income countries $(4.7 \%)[7,8]$. Some women may experience their first depressive episode during pregnancy, whereas others with a history of depression are at increased risk for its recurrence, continuation, or exacerbation $[9,10]$.

Depression in pregnancy is associated with adverse child outcomes and places women at greater risk for inadequate prenatal care, alcohol use, and poorer weight gain in 
pregnancy: each of these factors affects the unborn infant and slows fetal growth. The effects can also continue until adolescent time [11-13]. Most studies of maternal depression have focused on postnatal depression. However, depression is the most prevalent psychiatric disorder during pregnancy [14]. According to a recent systematic review of cohort studies, the prevalence of antenatal depression was $14 \%$ compared to a $10.5 \%$ pooled prevalence of postnatal depression. Most antenatal depression prevalence rates found in studies in developing countries were around 20\% [15, 16]. In Ethiopia, more than one in ten pregnant women and one in 20 postnatal women suffer from undetected depression and around half of them have thoughts of ending their life [17].

In Ethiopia, perinatal mental disorders contribute to maternal morbidity, in terms of poorer health, increased disability, and prolonged labor [18].

A study conducted in Turkey reported that depressive symptoms were found in $27(21.4 \%)$, for the women with no formal education. Women who were dissatisfied with their marriage $(38,14.5 \%)$ were more likely to be depressed than women who were satisfied $(33,8.5 \%)$. It was found that BDI scores were $>17$ in women who had no perceived support from a significant person (12, 24.0\%). Overall, 24 (24.2\%) women with unplanned pregnancy had a significantly higher rate of depression than the planned pregnancy groups $(47,8.5 \%)$. The regression analysis showed factors that affect $\mathrm{AD}$ as follows: lower education level of women, unplanned pregnancy, and lack of social support during pregnancy [19].

A cross-sectional study done in Tanzania shows that having a previous depressive episode ( $\mathrm{OR} 4.35, P<0.01$ ), low (OR 2.18, $P<0.01$ ) or moderate (OR 1.86, $P=0.04$ ) satisfaction with ability to access basic needs, conflicts with the current partner (OR 1.89, $P<0.01$ ), or booking earlier for ANC (OR 1.87, $P=0.02$ ) were independent predictors of antenatal depression in the logistic regression model [20]. According to the study done by using EPDS ( $>13$ cutoff point) in Addis Ababa, Ethiopia, antenatal depression was significantly higher among women who had not planned their current pregnancy. Those women who had not planned their current pregnancy were 2.78 times more likely to have antenatal depression than those who had planned their pregnancy $(\mathrm{AOR}=2.78$ (95\% CI: 1.59-4.85)). Absence of support from the baby's father was also associated with higher odds of having antenatal depression. Pregnant women who experienced lack of support from the baby's father had $89 \%$ higher odds of having antenatal depression when compared with women who got support from the baby's father $(\mathrm{AOR}=1.89$ (95\% CI: 1.06-3.36)).

History of depression was found to be one of the factors associated with antenatal depression. Those pregnant women who had previous history of depression were nearly three times at higher odds of having depression as compared to pregnant women who had no history of depression $(\mathrm{AOR}=2.57$ (95\% CI: 1.48-4.48)). Educational level, community's support, and partner's feeling on the current pregnancy were not significantly associated with depression during pregnancy in the multivariable logistic regression model [21].
The few prevalence studies among pregnant women in SSA suggest that the probable or definite psychiatric morbidity is high and also in Ethiopia. Perinatal mental disorders contribute to maternal morbidity. There is evidence that a number of risk factors are associated with maternal depression. Risk factors include the following: substance use, previous history of depression, age, poor marital relationships, low social status, poor social support, and unplanned or unwanted pregnancy $[19,22]$.

So far, few studies have been conducted to assess antenatal depression and associated factors in Ethiopia, but a study conducted in pastoralist community like Somali regional state and specifically Faafan zone is lacking. As Somali region has limited health service, a study which addresses the topic is much needed. This study attempted to examine the prevalence and factors associated with antenatal depression in Faafan zone, Somali regional state, Ethiopia.

The objective of this study was to assess the prevalence of depression and its associated factors among pregnant women attending ANC at health institutions of Faafan zone of Somali region, Eastern Ethiopia.

\section{Methods and Materials}

2.1. Study Design and Population. The study was conducted in Faafan zone of Ethiopian Somali region, Eastern Ethiopia, from January to September 2015. Ethiopian Somali regional state is located in the southeast part of the country. Somali region has nine administrative zones, namely, Faafan, Siti, Fik, Dhagahbur, Korrahe, Warder, Gode, Afder, and Liban. Faafan zone has 5 hospitals and 12 health centers. Maternal health services are free of charge in Ethiopia. ANC is provided in all selected health facilities [23]. ANC from a skilled provider is important to monitor pregnancy and reduce morbidity and mortality risks for the mother and child during pregnancy, delivery, and the postnatal period. According to Ethiopian Demographic and Health Survey (EDHS) of 2011, 34 percent of women who gave birth in the five years preceding the survey received ANC from a trained health professional at least once for their last birth. ANC utilization in Somali region was 21 percent. Institutional-based crosssectional study design was employed among 403 pregnant mothers attending at health institutions in Faafan zone. The study populations were selected pregnant women attending ANC at the selected health institutions of Faafan zone from January to September 2015.

2.2. Sample Size and Sampling Procedures. The sample size was calculated using the single population proportion formula with the assumption: proportion of antenatal depression to be $50 \%$ since we could not find previous research with the same setting in Ethiopia, 95\% certainty, 5\% for $d$, and $10 \%$ for nonresponse rate. Accordingly, the calculated sample size became 403 .

Institutions were stratified based on their service capacity into hospital and health centers. One hospital and seven health centers were selected randomly from all health facilities found in Faafan zone. The proportional sample size was allocated to selected health institutions based on their 
average daily ANC case flows. All consented ANC attendant women were taken into the study until the sample size was reached. Any apparently healthy pregnant women at any age of gestation who had come to the selected health institutions during the study period were included in the study. However, pregnant women who were critically ill, laboring, and unable to hear or speak during data collection were excluded from the study.

\subsection{Data Collection}

2.3.1. Instruments. The Geriatric Depression Scale (GDS) is an evaluation tool in the diagnosis of depression. It is validated with Cronbach's alpha 0.84, test-retest reliability 0.91 , and the concurrent validity of 0.83 to diagnose older adult's depression [24]. It is also validated with internal alpha 0.903 and test-retest reliability (ICC $=0.941$ (95\% CI: 0.886-0.970)) in Parkinson's disease [25]. EPDS has been also used to detect depressive symptoms [26]. The EPDS is a 10-item questionnaire, scored from 0 up to 3 (higher score indicating more depressive symptoms), that has been validated for detecting depression in antepartum and postpartum in many countries. The instrument was also validated in public health facilities in Ethiopia for postpartum use and showed sensitivity of $84.6 \%$ and specificity of $77.0 \%$ at the cutoff score 7/8 [22]. The cutoff point of EDPS among pregnant women is usually higher than postpartum women [27]. In Ethiopia, the EPDS cutoff point of 13 is used to identify pregnant women with depressive symptom [21]. We use EPDS for the purpose of this study.

Pregnant women who scored 13 and above were considered depressed women while pregnant women who scored below 13 were classified as nondepressed women [21]. Social support was measured using the Oslo-3 Social Support Scale (OSS-3) with three questions.

The response categories were assessed independently for each of the three questions, and a sum score was created by summarizing the raw scores.

The Oslo-3 scale has been used in several studies, thus confirming its feasibility and predictive validity with respect to psychological distress. In this study, the scale is used as both a sum score and an item-by-item scale.

The sum score scale ranging from 3 to 14 was used, which was then operationalized into three broad categories: "poor support" (3-8), "moderate support" (9-11), and "strong support" (12-14). A structured questionnaire was used to collect sociodemographic characteristics and clinical factors. Baseline clinical information was traced from the client's record by using checklists/data extraction formats.

2.4. Data Collection Tools and Procedures. The Somali version of adopted Edinburgh Postnatal Depression Scale which has ten items was used to collect data. A questionnaire adopted in English was translated to local Somali language by a language expert and then translated back to English to check consistency and accuracy. The Somali version of the questionnaire was used to collect data. Before conducting full study, a pretest was conducted among 21 clients (5\% of the sample size) attending in Lefaisa health center. All necessary modifications were made before the actual data collection. Data was collected by trained, experienced eight BSc nurses under the supportive supervision of principal investigators and two trained supervisors. A three-day training was given to the data collectors and supervisors on the data collection tool, interview technique, eligible study subjects, and consent. History of chronic illness was taken from medical records of the clients.

2.5. Data Processing and Analysis. First, the collected data was checked for completeness and consistency. Then, it was coded and entered in to Epi Info version 3.5.3. Then, data was exported and analyzed by using SPSS version 16 . Descriptive statistic was used to determine the prevalence of antenatal depression.

Binary logistic regressions were conducted between the predictors and antenatal depression. Using significant variables $(P<0.05)$ from binary logistic regression models, a multivariable logistic regression model was fitted to identify the independent predictors of antenatal depression. The strength of association was measured by odds ratios with $95 \%$ confidence intervals. All tests were two sided, and statistical significance was declared at $P<0.05$.

\subsection{Operational Definition}

(i) Depression. Women having pregnancy scores 13 out of 30 points were considered having antenatal depression

(ii) Clinical Factors. It is defined as gestational age, parity, maternal depression from previous pregnancy, previous history of depression and family history of depression, complications during current pregnancy, medication during pregnancy, previous poor pregnancy outcomes, and chronic medical illness

(iii) Substance Use. It is defined as current use when patients use a specified substance in the last 12 months

(iv) Psychosocial Factors. Social support is defined as "poor support" (a score of 3-8), "moderate support" (a score of 9-11), and "strong support" (a score of 12-14)

(v) Chronic Medical Illness. It is defined as cases such as diabetic mellitus, hypertension, gynecological disorders, lung disorders, and urinary tract infection

2.7. Ethical Consideration. Ethical clearance was obtained from the directorate of research and community service of Jigjiga University. Permission letter was taken from Somali Regional Health Bureau. Support letters were obtained from selected health institutions. Prior to data collection, written informed consent was obtained from the participants after a detailed explanation of the study and the study procedures. Only participants who gave written consent were interviewed in a separate room. Personal identifying details were not recorded. Participants identified with depressive 
symptoms were advised to visit the psychiatric clinic for better evaluation and treatment.

\section{Results}

3.1. Sociodemographic Characteristics. Three hundred ninetyfive respondents participated in this study making the response rate of $98 \%$. The mean age of study participants was 25.71 years $(S D \pm 5.04)$ with the age range of 15 to 40 years. Three hundred twenty-one $(81.3 \%)$ participants were married and living together with their husband. Two hundred eight $(52.7 \%)$ of study participants were illiterate, and more than eighty percent of study subjects were urban residents (Table 1).

3.2. Obstetric and Clinical Characteristics. Most of the study participants $(70.9 \%)$ were in the second and third trimester at the time of the study. One hundred nine $(27.6 \%)$ study participants had chronic medical illness. One hundred fifty-nine $(39.5 \%)$ participants were taking medication during the study period, and 29 (7.3\%) participants had developed complication during current pregnancy. One-fifth of the pregnant women $(84,21.3 \%)$ had experienced previous poor pregnancy outcome. Majority $(357,90.4 \%)$ of the respondents reported that their current pregnancy was planned/wanted (Table 2).

3.3. Psychiatric History, Social Support, and Substance Use. The study revealed that $41(10.4 \%)$ of the study participants had a previous history of depression, and 19 (4.8\%) of the study participants reported presence of history of depression in their family. Fifty-one (12.9\%) participants had poor social support. Among the respondents, 44 (11.1\%) had used Khat while $17(4.3 \%)$ had used tobacco product at least once in the last twelve months. The percent of pregnant women who had used alcohol and cannabis at least one time in the last twelve months during the study was $2(0.5 \%)$ and 1 $(0.3 \%)$, respectively (Table 3 ).

3.4. Prevalence of Antenatal Depression. The internal consistency of the EPDS tool was acceptable (Cronbach's $\alpha=0.77$ ). The study revealed that the overall prevalence of antenatal depression in this study was $24.3 \%$.

3.5. Factors Associated with Antenatal Depression. Factors associated with antenatal depression were identified by using logistic regression. During bivariate logistic regression, variables like residence, monthly income, marital status, education, current pregnancy complication, chronic medical illness, number of pregnancy, pregnancy status, family history of depression, previous history of depression, previous history of antenatal depression, social support, and Khat use were associated with antenatal depression. However, factors like age, occupation, duration of pregnancy, previous poor pregnancy outcome, alcohol use, tobacco use, and cannabis use were not associated with antenatal depression. Multilogistic regression revealed that mothers who were married but not living together with their partners were three times more likely to get antenatal depression than those mothers who are married and living together with their husbands (AOR 2.91 (95\% CI: 1.36, 6.23)).
TABLE 1: Distribution of pregnant women by sociodemographic factors attending ANC at health institutions of Faafan zone, December $2015(n=395)$.

\begin{tabular}{|c|c|c|c|}
\hline Variables & Categories & Frequencies & Percent (\%) \\
\hline \multirow{4}{*}{ Age } & $15-24$ & 150 & 38.0 \\
\hline & $25-34$ & 218 & 55.2 \\
\hline & $35-44$ & 27 & 6.8 \\
\hline & Total & 395 & 100.0 \\
\hline \multirow{3}{*}{ Residence } & Urban & 330 & 83.5 \\
\hline & Rural & 65 & 16.5 \\
\hline & Total & 395 & 100.0 \\
\hline \multirow{3}{*}{ Religion } & Muslim & 351 & 88.9 \\
\hline & Orthodox & 32 & 8.1 \\
\hline & Protestant & 12 & 3.0 \\
\hline \multirow{5}{*}{$\begin{array}{l}\text { Marital } \\
\text { status }\end{array}$} & $\begin{array}{l}\text { Married and living } \\
\text { together }\end{array}$ & 321 & 81.3 \\
\hline & $\begin{array}{l}\text { Married but not living } \\
\text { together }\end{array}$ & 50 & 12.7 \\
\hline & Single & 6 & 1.5 \\
\hline & Divorced & 14 & 3.5 \\
\hline & Widowed & 4 & 1.0 \\
\hline \multirow{4}{*}{ Ethnicity } & Somali & 272 & 68.9 \\
\hline & Oromo & 62 & 15.7 \\
\hline & Amhara & 36 & 9.1 \\
\hline & Gurage/Tigre & 25 & 6.3 \\
\hline \multirow{3}{*}{ Education } & Illiterate & 208 & 52.7 \\
\hline & Primary education & 112 & 28.3 \\
\hline & $\begin{array}{l}\text { Secondary education and } \\
\text { above }\end{array}$ & 75 & 19.0 \\
\hline \multirow{5}{*}{ Occupation } & Housewife & 219 & 55.4 \\
\hline & Merchant & 54 & 13.7 \\
\hline & Farmer & 41 & 10.4 \\
\hline & Employee & 67 & 17.0 \\
\hline & Student & 14 & 3.5 \\
\hline
\end{tabular}

Those women who had chronic medical illness were two times more likely to have antenatal depression than those who had no chronic depression $(\mathrm{AOR}=2.21$ (95\% CI: 1.09-4.45)). Absence of social support was also associated with higher odds of having antenatal depression when compared with women who had social support $(\mathrm{AOR}=3.34$ (95\% CI: 1.50-7.43)). Pregnant mothers who had previous history of depression were six times at higher odds of having antenatal depression as compared to pregnant women who had no history of depression $(\mathrm{AOR}=6.02$ (95\% CI: $2.31-$ 10.01)). Educational level, number of pregnancy, and previous history of depression were also factors associated with antenatal depression (Table 4).

\section{Discussion}

This study identified sociodemographic factors, obstetric factors, clinical factors, social support factor, and substance 
TABLE 2: Obstetric and clinical characteristics of pregnant women attending ANC at health institutions of Faafan zone, December $2015(n=395)$.

\begin{tabular}{|c|c|c|c|}
\hline Variables & Categories & Frequencies & Percent (\%) \\
\hline \multirow{3}{*}{$\begin{array}{l}\text { Chronic medical } \\
\text { illness }\end{array}$} & Yes & 109 & 27.6 \\
\hline & No & 286 & 72.4 \\
\hline & Total & 395 & 100.0 \\
\hline \multirow{6}{*}{ Number of pregnancy } & First & 77 & 19.5 \\
\hline & Second & 120 & 30.4 \\
\hline & Third & 90 & 22.8 \\
\hline & Fourth & 32 & 8.1 \\
\hline & $\begin{array}{l}\text { Fifth and } \\
\text { above }\end{array}$ & 76 & 19.2 \\
\hline & Total & 395 & 100.0 \\
\hline \multirow{3}{*}{ Pregnancy status } & $\begin{array}{c}\text { Planned and } \\
\text { wanted }\end{array}$ & 357 & 90.4 \\
\hline & $\begin{array}{l}\text { Unplanned or } \\
\text { unwanted }\end{array}$ & 38 & 9.6 \\
\hline & Total & 395 & 100.0 \\
\hline \multirow{3}{*}{$\begin{array}{l}\text { Previous poor } \\
\text { pregnancy outcome }\end{array}$} & Yes & 84 & 21.3 \\
\hline & No & 311 & 78.7 \\
\hline & Total & 395 & 100.0 \\
\hline \multirow{3}{*}{$\begin{array}{l}\text { Complication during } \\
\text { current pregnancy }\end{array}$} & Yes & 29 & 7.3 \\
\hline & No & 366 & 92.7 \\
\hline & Total & 395 & 100.0 \\
\hline \multirow{4}{*}{ Duration of pregnancy } & First TM & 115 & 29.1 \\
\hline & Second TM & 139 & 35.2 \\
\hline & Third TM & 141 & 35.7 \\
\hline & Total & 395 & 100.0 \\
\hline
\end{tabular}

use factors that were associated with antenatal depression. The study revealed that the overall prevalence of antenatal depression was 24.3\%. Marital status, educational status, chronic medical illness, number of pregnancy, previous depression history, and social support were factors associated with antenatal depression.

The level of antenatal depression found in this study is relatively lower as compared to findings of studies done in Addis Ababa, Ethiopia, that revealed 24.94\% prevalence of antennal depression [28]. Even though there is no tangible evidence for this difference, the difference in the sample size of studies and sociodemographic characteristics may contribute for this variation. Similarly, the prevalence of antenatal depression in this study is lower than the finding of the study conducted in other parts of Africa such as Cape Town and Tanzania which reported the prevalence of 39\% and $39.5 \%$, respectively $[20,29]$. However, the finding of this study is higher than the finding of other studies done in different parts of the world such as $4.4 \%$ in China [30] and $18.3 \%$ in Bangladesh [31]. These variations may be attributed to differences in sociodemographic and obstetric factors. Different studies conducted in various parts of the globe revealed that the impact of factors such as educational
TABLE 3: Psychiatry, social support, and substance use of pregnant women attending ANC at health institutions of Faafan zone, December $2015(n=395)$.

\begin{tabular}{|c|c|c|c|}
\hline Variable & Categories & Frequencies & Percent (\%) \\
\hline \multirow{3}{*}{$\begin{array}{l}\text { Previous depression } \\
\text { history }\end{array}$} & Yes & 41 & 10.4 \\
\hline & No & 354 & 89.6 \\
\hline & Total & 395 & 100.0 \\
\hline \multirow{4}{*}{ Social support } & Poor & 51 & 12.9 \\
\hline & Moderate & 146 & 37.0 \\
\hline & Strong & 198 & 50.1 \\
\hline & Total & 395 & 100.0 \\
\hline \multirow{3}{*}{$\begin{array}{l}\text { Family history of } \\
\text { depression }\end{array}$} & Yes & 19 & 4.8 \\
\hline & No & 376 & 95.2 \\
\hline & Total & 395 & 100.0 \\
\hline \multirow{3}{*}{ Khat use } & Yes & 44 & 11.1 \\
\hline & No & 351 & 88.9 \\
\hline & Total & 395 & 100.0 \\
\hline \multirow{3}{*}{ Tobacco use } & Yes & 17 & 4.3 \\
\hline & No & 378 & 95.7 \\
\hline & Total & 395 & 100.0 \\
\hline \multirow{3}{*}{ Alcohol use } & Yes & 2 & .5 \\
\hline & No & 393 & 99.5 \\
\hline & Total & 395 & 100.0 \\
\hline \multirow{3}{*}{ Cannabis use } & Yes & 1 & .3 \\
\hline & No & 394 & 99.7 \\
\hline & Total & 395 & 100.0 \\
\hline
\end{tabular}

status and pregnancy planning affects the level of antenatal depression. The difference in antenatal depression in this study and study conducted in Addis Ababa may be attributed to the difference in the contraceptive prevalence rate. For instance, the contraceptive prevalence rate in Addis Ababa town (62.5\%) is higher than that of Somali region (4.1\%) [27].

Studies have shown that chronic medical illness and previous history of antenatal depression factors are associated with antenatal depression in Ethiopia and other parts of the world [32]. Similarly, this study revealed that the odds of antenatal depression were two times among the mother who had chronic medical illness during the study. This is similar with the studies conducted in Durban, Rio de Janeiro, Brazil [28, 33]. Previous history of depression was the largest antenatal depression contributor in this study.

In this study, previous history of depression was significantly associated with antenatal depression. This finding is similar with a study conducted in Addis Ababa which reported a significant association between previous history of depression and antenatal depression [32].

This study indicated that social support is also another factor associated with antenatal depression. The finding of this study is similar with the study conducted in Addis Ababa which showed that the odd of developing antenatal depression was $89 \%$ higher in those pregnant women who 
TABLE 4: Predictors of antenatal depression among pregnant women attending ANC at health institutions of Faafan zone, December 2015 $(n=395)$.

\begin{tabular}{|c|c|c|c|c|}
\hline \multirow{2}{*}{ Variable } & & \multicolumn{2}{|c|}{ Antenatal depression } & \multirow[t]{2}{*}{ AOR (95\% CI) } \\
\hline & & Yes $=96$ & $\mathrm{No}=299$ & \\
\hline \multirow{4}{*}{ Marital status } & Married and living together & $64(66.7 \%)$ & $257(86.0 \%)$ & 1.0 \\
\hline & Married but not living together & $19(19.8 \%)$ & $31(10.4 \%)$ & $2.91(1.36,6.23)$ \\
\hline & Divorced and single & $11(11.4 \%)$ & $9(\%)$ & $3.21(0.93,11.02)$ \\
\hline & Widowed & $2(2.1 \%)$ & $2(.7 \%)$ & $1.99(0.18,3.46)$ \\
\hline \multirow{3}{*}{ Educational status } & Illiterate & $54(56.2 \%)$ & $154(51.5 \%)$ & $2.49(1.09,5.71)$ \\
\hline & Primary & $30(31.2 \%)$ & $82(27.4 \%)$ & $1.80(0.75,4.37)$ \\
\hline & Secondary and above & $12(12.5 \%)$ & $63(21.1 \%)$ & 1.0 \\
\hline \multirow{2}{*}{ Chronic medical illness } & Yes & $21(21.9 \%)$ & $88(29.4 \%)$ & $2.21(1.09,4.45)$ \\
\hline & No & $75(78.1 \%)$ & $211(70.6 \%)$ & 1.0 \\
\hline \multirow{5}{*}{ Number of pregnancy } & First & $26(27.1 \%)$ & $51(17.1 \%)$ & $8.89(2.09,12.78)$ \\
\hline & Second & $30(31.2 \%)$ & $90(30.1 \%)$ & $7.32(1.81,11.97)$ \\
\hline & Third & $20(20.8 \%)$ & $70(23.4 \%)$ & $5.77(1.38,9.41)$ \\
\hline & Fourth & $6(6.2 \%)$ & $26(8.7 \%)$ & $4.73(0.89,7.49)$ \\
\hline & Fifth and above & $14(14.6 \%)$ & $62(20.7 \%)$ & 1.0 \\
\hline \multirow{2}{*}{ Previous depression history } & Yes & $31(32.3 \%)$ & $10(3.3 \%)$ & $6.02(2.31,10.01)$ \\
\hline & No & $161(67.7 \%)$ & $295(96.7 \%)$ & 1.0 \\
\hline \multirow{3}{*}{ Social support } & Poor & $28(29.2 \%)$ & $23(7.7 \%)$ & $3.34(1.50,7.43)$ \\
\hline & Moderate & $35(36.5 \%)$ & $111(37.1 \%)$ & .990 \\
\hline & Strong & $33(34.4 \%)$ & $166(55.2 \%)$ & 1.0 \\
\hline
\end{tabular}

experienced lack of baby's father support [32]. The possible reason may be women who have social support during pregnancy may have low stress compared to women with poor social support. This study also found that the odds of antenatal depression among mothers who cannot read and write were two times higher than mothers who have some level of education. This is similar with other studies conducted in Turkey which showed that lower education level of women was a factor that affects antenatal depression [19]. This may due to the reason that educated mothers have higher access to reproductive health information, and this in turn may reduce stress during pregnancy.

\section{Limitation of the Study}

The study did not comprehensively address the possible factors associated with antenatal depression. The direct translation of EPDS was used but the psychometric properties of the tools were not validated in Ethiopia. Data for chronic medical illness was taken from records so that there is a possibility to make bias due to the nature of secondary data.

\section{Conclusion and Recommendations}

The study has assessed the prevalence of antenatal depression and associated factors. The study revealed that onefifth of study population had antenatal depression. Marital status, educational status, chronic medical illness, number of pregnancy, previous depression history, and social support were factors associated with antenatal depression.
Ethiopia Federal Ministry of Health and Somali Regional Health Bureau should work very hard to create awareness about the importance of contraceptive use in the study area. Health education should be given to the community on the importance of pregnancy planning and social support during pregnancy at all levels. Ethiopian Ministry of Health and Somali Regional Education Bureau should work cooperatively to boost women's educational status. ANC providers at all levels should provide counseling, medical care, and emotional support to pregnant women.

\section{Abbreviations}

ANC: Antenatal care

AD: Antenatal depression

AOR: Adjusted odds ratio

BDI: Becky depression inventory

CI: Confidence interval

EDHS: Ethiopian Demographic and Health Survey

EPDS: Edinburgh Postnatal Depression Scale

OR: Odds ratio

OSS-3: Oslo-3 Social Support Scale

SD: $\quad$ Standard deviation

SPSS: Software Package for Social Science

SSA: Sub-Saharan Africa.

\section{Data Availability}

Data is available on the hand of the authors. 


\section{Ethical Approval}

Ethical clearance was obtained from the Institutional Review Board of Jigjiga University, Directorate of Research, Publication and Technology Transfer. The right of the respondents to withdraw from the interview was assured. Any personal identifier was not encoded; identifiers of the women were replaced with identification numbers. The members of Ethics Committee are Mr. Adugna Nigussie, Mr. Wubareg Seifu, and Mr. Lemma Mideksa.

\section{Consent}

Informed verbal consent was obtained from each woman after the purposes of the study were explained to them.

\section{Disclosure}

The grant agency has no role in the design of the study, collection, analysis, and interpretation of data and in writing the manuscript. The university only needs the final report which is submitted by the research team.

\section{Conflicts of Interest}

The authors declare that they have no any competing interests.

\section{Authors' Contributions}

ET and MK had taken a principal role in the conception of ideas, developing methodologies, data collection, analyses, and write up of the article. GT has taken a role in the preparation of this manuscript. All authors read and approved the final manuscript.

\section{Acknowledgments}

We forward our heartfelt gratitude to Jigjiga University for its financial support. We also thank our study participants and health office managers of different levels for their cooperation. Finally, we are indebted to the data collectors and supervisors for their valuable time.

\section{References}

[1] B. M. Leung and B. J. Kaplan, "Perinatal Depression: Prevalence, Risks, and the Nutrition Link-A Review of the Literature," Journal of the American Dietetic Association, vol. 109, no. 9, pp. 1566-1575, 2009.

[2] WHO, Depression and other common mental disorders: global health estimates, world health organization, 2017.

[3] D. A. Fekadu, E. R. Miller, and L. Mwanri, "Antenatal depression and its association with adverse birth outcomes in low and middle income countries: a systematic review and meta analysis," PLos One, vol. 15, no. 1, 2020.

[4] B. N. Gaynes, N. Gavin, S. Meltzer-Brody et al., "Perinatal depression: prevalence, screening accuracy, and screening outcomes, Agency for Healthcare Research and Quality," Evidence Report/Technology Assessment, vol. 119, 2005.
[5] E. R. Declerq, C. Sakala, and M. P. Corry, Listening to mothersreport of the first national U.S. survey of women's childbearing experiences, Maternity Center Association, New York, 2002.

[6] World Health Organization, The global burden of disease 2004 update Geneva, World Health Organization, 2009.

[7] Federal Ministry of Health, Introducing Ethiopia's National Mental Health Strategy, TSEHaAI, Ethiopia, 2012.

[8] C. D. Mathers and D. Loncar, "Projections of global mortality and burden of disease from 2002 to 2030," Public Library of Science Medicine, vol. 3, 2006.

[9] R. Nonacs and L. S. Cohen, "Depression during pregnancydiagnosis and treatment options," Journal of clinical Psychiatry, vol. 63, no. 7, pp. 24-30, 2002.

[10] V. K. Burt and K. Stein, "Epidemiology of depression throughout the female life cycle," Journal of Clinical Psychiatry, vol. 63, 2002.

[11] T. D. Wachs, M. M. Black, and P. L. Engle, "Maternal Depression: A Global Threat to Children's Health, Development, and Behavior and to Human Rights," Child Development Perspectives., vol. 3, no. 1, pp. 51-59, 2009.

[12] T. Diego, M. H.-R. Field, C. K. SSchanberg, and V. H. Gonzalez-Quintero, "Prenatal depression restricts fetal growth," Early Human Development, vol. 85, pp. 65-70, 2008.

[13] S. Pawl By, D. Hay, D. Sharp, C. Waters, and V. O. Keane, "Antenatal depression predicts depression in adolescent offspring: Prospective longitudinal community-based study," Journal of Affective Disorders, vol. 113, no. 3, pp. 236-243, 2009.

[14] H. A. Bennett, A. Einarson, G. Koren, and T. R. Einarson, "Prevalence of depression during Pregnancy: systematic review," Obstetrics and Gynecology, vol. 103, no. 4, pp. 698709, 2004.

[15] P. Maullik, V. Patel, and J. Langham, "Depression and motherhood: when does it start?-In Abstracts of the XIII World Congress of Psychiatry," Cairo, World Psychiatric Association, vol. 114, 2005.

[16] K. Pereira, G. M. Lovisi, and L. A. Lima, "Depression during Pregnancy-Review of Epidemiological and Clinical Aspects in Developed and Developing Countries," Federal University of Rio de Janeiro Brazil, vol. 6, 2010.

[17] C. Hanlon, G. Medhin, A. Alem et al., "Sociocultural practices in Ethiopia: association with onset and persistence of postnatal common mental disorders," British Journal of Psychiatry., vol. 197, no. 6, pp. 468-475, 2010.

[18] C. Hanlon, G. Medhin, A. Alem et al., "Impact of antenatal common mental disorders upon perinatal outcomes in Ethiopia: the P-MaMiE population-based cohort study," Tropical Medicine \& International Health, vol. 14, no. 2, pp. 156-166, 2009.

[19] S. Yanikkerem and S. Ay, "Antenatal depression- prevalence and risk factors in a hospital based Turkish sample," J Pak Med Assoc, vol. 63, no. 4, 2013.

[20] S. F. Kaaya, J. K. Mbwambo, G. P. Kilonzo et al., "Socio-economic and partner relationship factors associated with antenatal depressive morbidity among pregnant women in," Tanzania Journal of Health Research, vol. 12, no. 1, pp. 2335, 2010.

[21] Y. Dibaba, M. Fantahun, and M. J. Hindin, "The association of unwanted pregnancy and social support with depressive symptoms in pregnancy: evidence from rural Southwestern Ethiopia," BMC Pregnancy and Childbirth, vol. 13, no. 1, 2013. 
[22] M. Tesfaye, C. Hanlon, D. Wondimagegn, and A. Alem, "Detecting postnatal common mental disorders in Addis Ababa, Ethiopia: Validation of the Edinburgh Postnatal Depression Scale and Kessler Scales," Journal of Affective Disorders, vol. 122, no. 1-2, 2010.

[23] Ethiopian central statistical Ethiopian demographic and health survey, CSA, 2011.

[24] G. Galeoto, J. Sansoni, M. Scuccimarri et al., "An evaluation of the psychometric properties of the Italian version of the geriatric depression scale," Depression Research and Treatment, vol. 2018, Article ID 1797536, 2018.

[25] P. Massai, F. Colalelli, J. Sansoni et al., "Galeoto G Reliability and validity of the geriatric depression scale in Italian subjects with Parkinson's disease," Parkinson's Disease, vol. 2018, article 7347859, 2018.

[26] D. Murray and J. L. Cox, "Screening for depression during pregnancy with EPDS (EPDS)," Journal of Reproductive and Infant Psychology, vol. 8, pp. 99-106, 1990.

[27] J. Gibson, K. McKenzie-McHarg, J. Shakespeare, J. Price, and R. Gray, "A systematic review of studies validating agency, EPDS in antepartum and postpartum women," Acta Psychiatrica Scandinavica, vol. 119, no. 3, pp. 50-64, 2009.

[28] J. G. Katon, J. Russo, A. R. Gavin, J. L. Melville, and W. J. Katon, "Diabetes and depression in pregnancy: is there an association?," Journal of Women's Health, vol. 20, 2011.

[29] M. Hartley, M. Tomlinson, E. Greco et al., "Depressed mood in pregnancy: prevalence and correlates in two Cape Town periurban settlements," Reproductive Health, vol. 8, no. 1, 2011.

[30] D. T. S. Lee, S. S. M. Chan, D. S. Sahota, A. S. K. Yip, M. Tsui, and T. K. H. Chung, "A prevalence study of antenatal depression among Chinese women," Journal of Affective Disorders, vol. 82, no. 1, pp. 93-99, 2004.

[31] H. E. Nasreen, Z. N. Kabir, Y. Forsell, and M. Edhborg, "Prevalence and associated factors of depressive and anxiety symptoms during pregnancy: a population based study in rural Bangladesh," BMC Women's Health, vol. 11, no. 1, 2011.

[32] A. Biratu and D. Haile, "Prevalence of antenatal depression and associated factors among pregnant women in Addis Ababa, Ethiopia," Reproductive Health, vol. 12, 2015.

[33] P. K. Pereira, G. M. Lovisi, D. L. Pilowsky, L. A. Lima, and L. F. Legay, "Depression during pregnancy: prevalence and risk factors among women attending a public health clinic in Rio de Janeiro, Brazil," Saúde Pública, vol. 25, no. 12, pp. 27252736, 2009. 